\title{
3ur Beadtung:
}

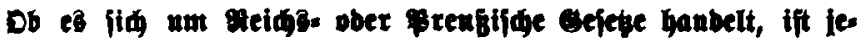

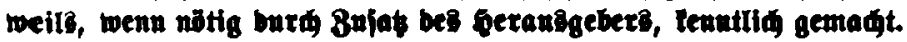

Baragraphenllberfinriften in edigen alammern fammen bom ecraugeber, foldie in runben slammern ans bem amtitifen rest.

\section{Inbaltouberfiujt.}

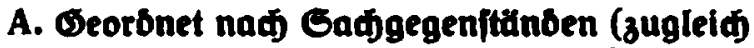 naథ set Folge bet Nummern).}

I. Berfafinugen.

1. Reldgverfaffung. Bom 11. Ruguft 1919...

1a. Reidsobefaffung. Bom 16. Qpptl 1871 . . . .

1b. Rufruf bes Bates ber Bollabeauftragten. Bom 12. Robember 1918

Selte

$1-17$

17-73

73-74

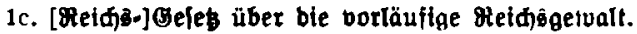
Bom 10. छebruar $1919 \ldots \ldots$

1d. [Reidbo.] abergangägeleb. Bom 4. Dlär 1919.

8. Brentidie Betfafiung. Bom 30. Robember 1980

2a. Breubifde Berfafiungiurtunbe. Bom 31. Yanuar 1850

3. [ReidS-] Rewublitfdukgefet. Bom 25. Măt 1930

4. [Peid3.] Berorbnung über bie Teutihen Glaggen. Bom 11. Ppril 1921/5. Plat 1926

$97-117$

$117-122$

$122-125$

II. Cheieqgebenbe Drgane unb Meiekgebung.

1. Reidistag unb Ianbtag:

5. Meidabwahlgefę. Bom 6. פlär 1924 . . . . 126-139

5a. Breub. Bahloefes [Ranbesmahloefey]. Bom 28. Oltober $1984 \ldots \ldots . \ldots . . .126-139$

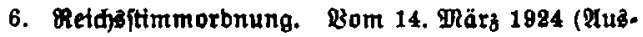
zug) $139-147$

7. [Reidor] Befeb über bie Beftebung ber Ge.

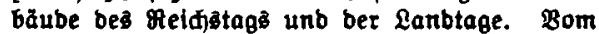
8. Mlai $1920 \ldots \ldots$. . . . . . . . 147-148

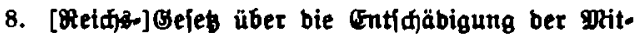
glteber be MeidatagB. Bom 15. Desember 1930

9. Befđjăftżorbmung für ben Reiđątag. Bom 12. Dezember 1922/31. Mắz $1931 \ldots$. . . . . 150-174 


\section{Intalt Büberfint.}

2. Meiđisat unb Stnatzirat:

10. [Breub.] Gefeb über bie Beftellung von $\mathfrak{D}$ it: gliebem bes Reichatats burd bie Rrobingialvermaltungen. Bom 3. 3uni 1921 ...... .

11. [Breub.] Befeb über bie æahlen zum Etaatzrat. Bom 16. Tezember 1920

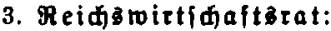

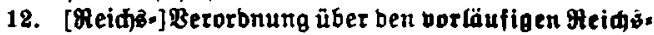

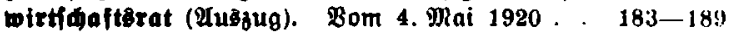

4. Geịeggebung:

13. [Meid)] Seieb über ber Boltsentficib. Rom

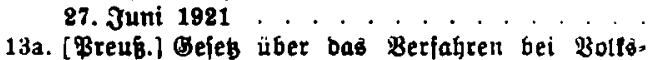
begehren unb Boltsentficheiben. Bomt 8. Jamuar 1926 . . . . . . . . . . . . . . . . . . .

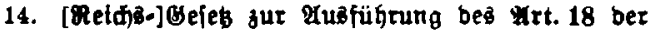

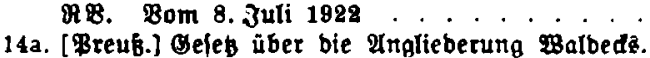
Bom 25. Juft 1928 'ं $\cdot \cdot \cdot \cdot \cdot \cdot \cdot \cdot \cdot \cdot \cdot \cdot$

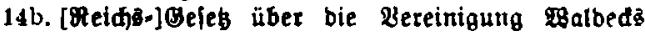
mit Breufen. Bom 7. Dezember 1928 ....

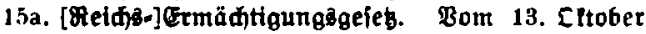
1983

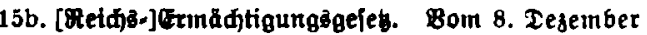
1883

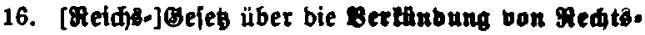
berorbunugen. Bom 13. Dttober 1983 . . . . 219-220

16a. [\$reub.] Oefeł über ble Bertünbung von Hed)tร. berorbrungen. Bom 9. घuguit 1924

17a. Radwetjung ber Berorbnungen auf Brumb von Irt 48 in ben Yahren 1919-1931 . . . . . .

17b. Inbaltsaberifiten ber Degember-Rotverorbnung 1930 unb bet 3uni-9lotberorbnung 1931

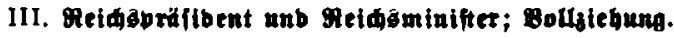

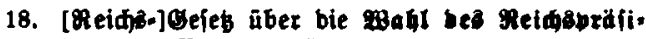
benten. Bom 6. פlărb 1984. . . . . . . . . 225-220

19. Steidsominiftergefę. Bom 27. Măr 1930 . . 287--235

20. Beiđăftsorbrung ber Meițżegienung. Bom 3. Mai 1924 . . . . . . . . . . . . . . . . $235-244$

\section{Berfaffungagexíntobarteit.}

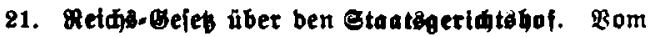
9. ЭuIi 1921 


\section{galyultoibierfist.}

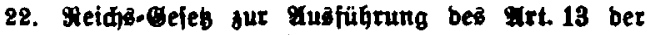

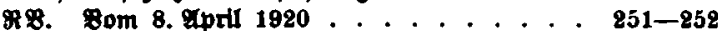

\section{Heamtenredt.}

23. Metobreantengefer. Bom 17. Mai 1907. .

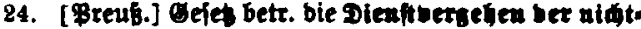
riditeritiden seanten. Bour 81. Sult 1868 .

25. Reidjbelolbungagefeb. Bom 16. Desember 1927

25a. Preus. Bejolbungageleb. Bom 17. Dezember 1887

253-295

286-317

$317-392$

$333-334$

VI. Hetrmadt.

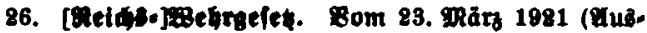
348)

VII. Finamsen.

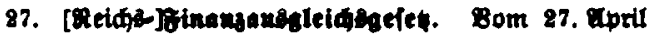
1986 (Guosug) . . . . . . . . . . . . . . .

28. [Reid-] Befteuerungogefeb. Bom 10. Auguft 1985

28a. [Retho] Cefes tber bte \$aufbalierumg ber Bertoaltumglofteraufgilfe. Bom 17. Jult 1990 . .

89. Rethobanghaltiorbuna, Bom 31. Dezember 1988/14. Toril 1930. . . . . . . . . . . . .

29a. [\$reub.] Sefeb betr. ben Staatboubbalt [Romp.

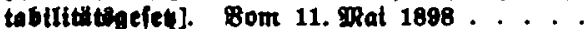

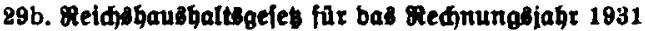

89c. Breub. Gaubhal tsoeleb fihr bas Bedmungsjahr 1981

$363-402$

403-417

$417-483$

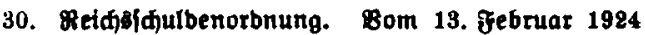

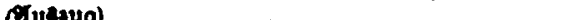

\section{Der Gtaatoburger.}

31. Beids. unb Gtantangehörigleitageter. Bom 28. 3uli 1913

38. [Reido-jetfonenftanbagefek. Bom 6. fुebruar 1875 (Autug) . . . . . . . . . . . . . . .

33. [Ret๘)-Breiatgigletigefes. Bom 1. Nobember 1867

33a. [\$reuв.] Befes über bie \&ufnahme neuanzie. benber Berfonen. Bom 31. Desember 1842

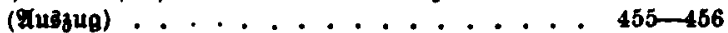

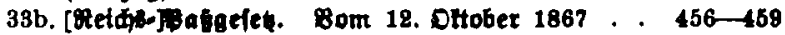

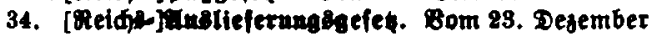
1989 (4u\&ug) 


\section{3nhaltoriberfint.}

35. [\$reub.] Bejes über bie \&ufbebung ber etanbes. vorredte bea abels. Bom 23. Juni 1920 . .

35.. [Preuß.] Berorbnung betr. bie Anberung ber Familiennamen. Bom 3. Robember 1919 . . 472-474

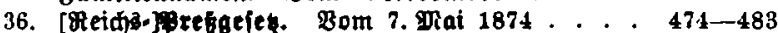

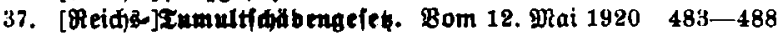

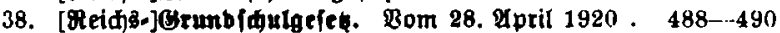

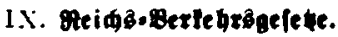

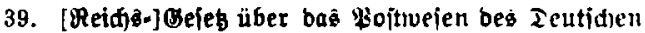
Metđ [Meidapoftgefé)]. Bom 28. Dttober 1871

39a. Reiథŝpoftfinanzgefę. Bom 18. Rã̃ 1924

40. [Reids]'Beleb betr. ben Staatöpertrag über ben abergang ber Staatşeifenbahnen auf baş Beid). Bom 30. Ypril 1920

11. [Reidja.] (Seleb über bie Ieutiat)e Keidrsbahngeiell:

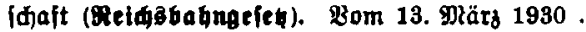

42. [Reid)B.] (Sejeb betr. ben Etaat Boertrag über ben

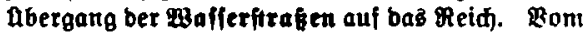
29. Juli 1921

43. [Reido] \&nftueriehrogefes. Bom 1. Auguft 1922 (भนติวนด)

\section{x. Btantstirdenredt.}

44. [\$reub.] Befes betr. ben Bertrng mit ben Goat" gelifłen Lambestirdien. Bom 26. Juni 1931.

45. [Breub.] Seles betr. ben Bertrag mit bem \$eiligen Etuhl. Rom 3. Yuguft 1929 . . . . 560. 567

\section{Uus bem Gemerberedt.}

46. Cetmetbeorbnung für bas ieutiche geid. Bont 21. Iuni 1869 ............. 568-718

47. [Meid)- Saftithtengeles. Wom 88. Qiptil 1930, 714-730 
Inbaltsüberfidt.

B. Beorónet nad ber 3eitfolge.

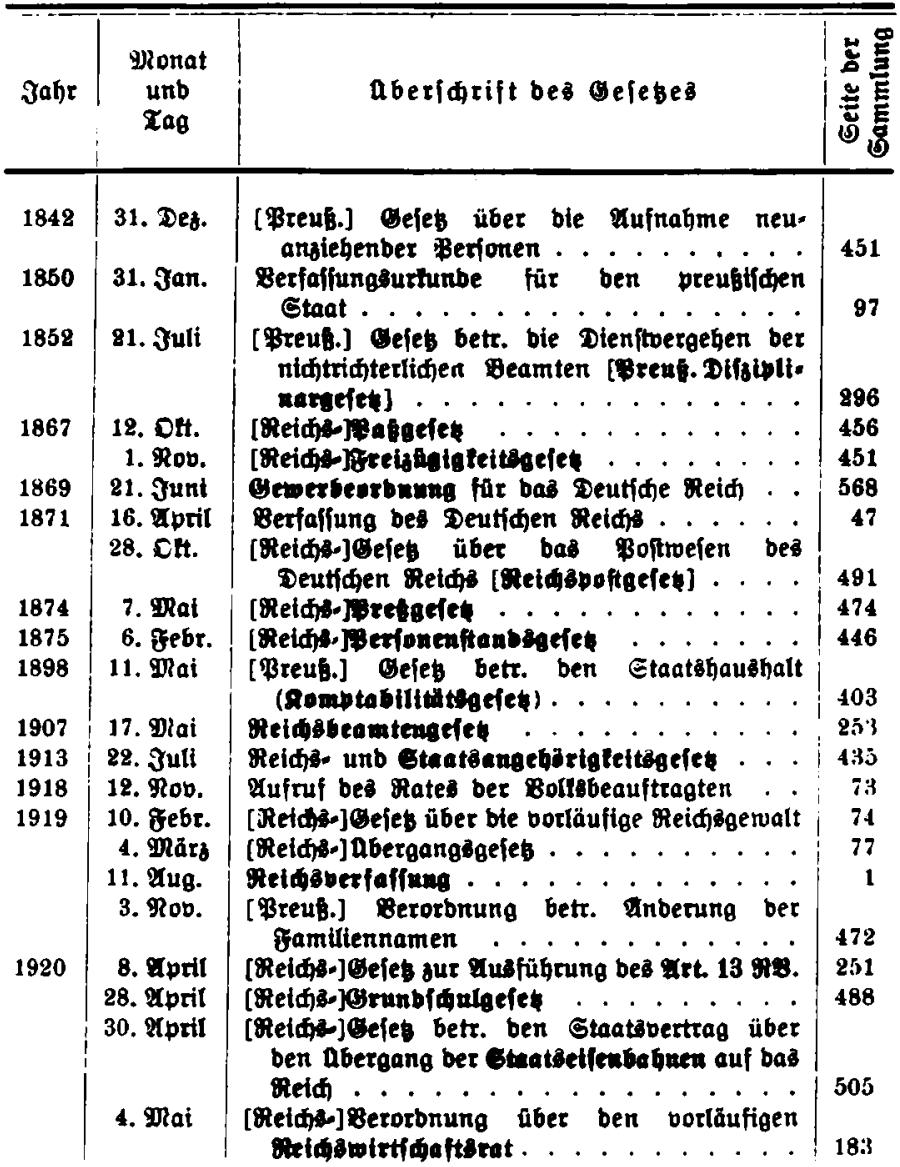




\section{Inlyeltoüberfist.}

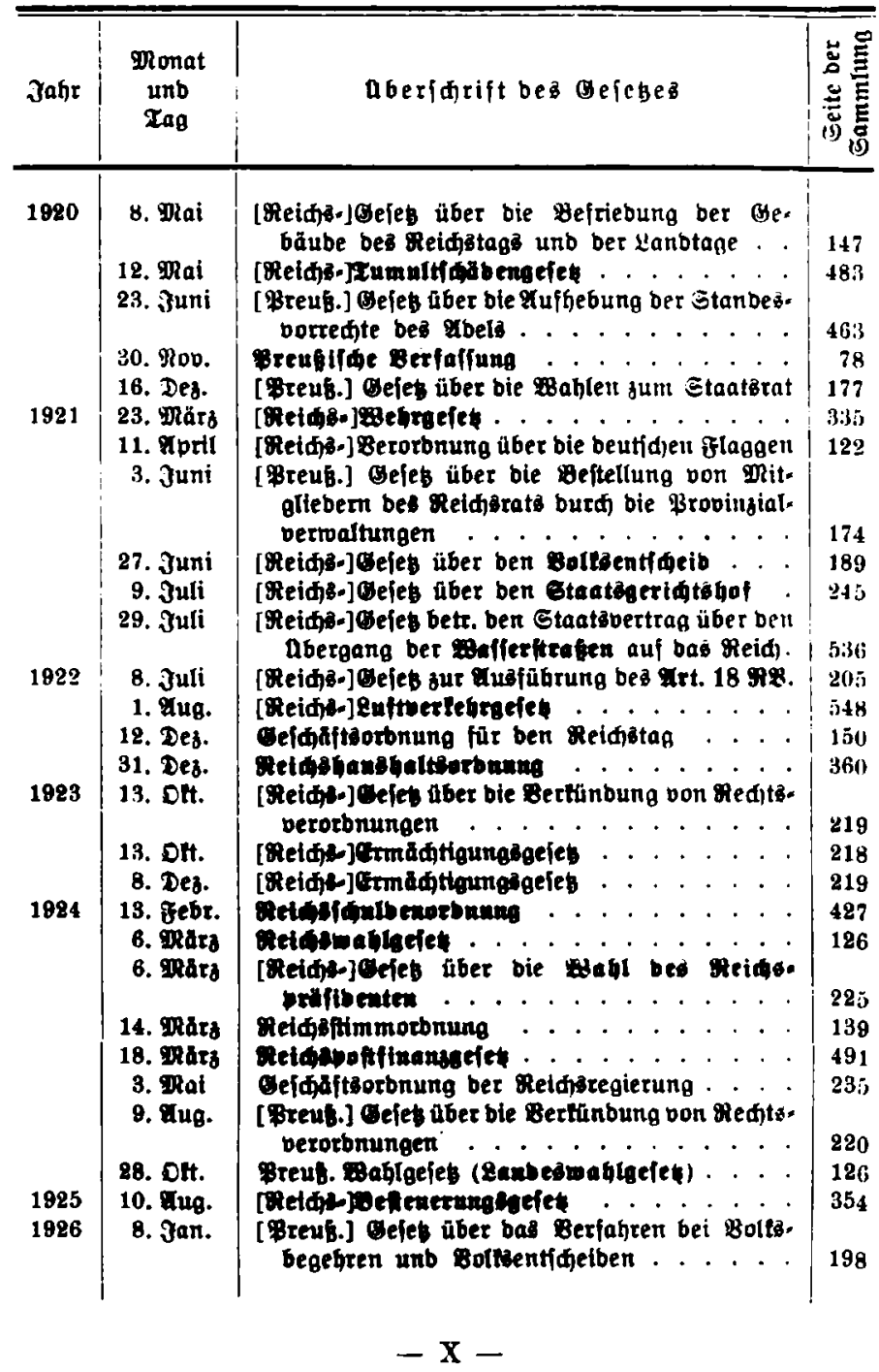


Juyaltbïberfidt.

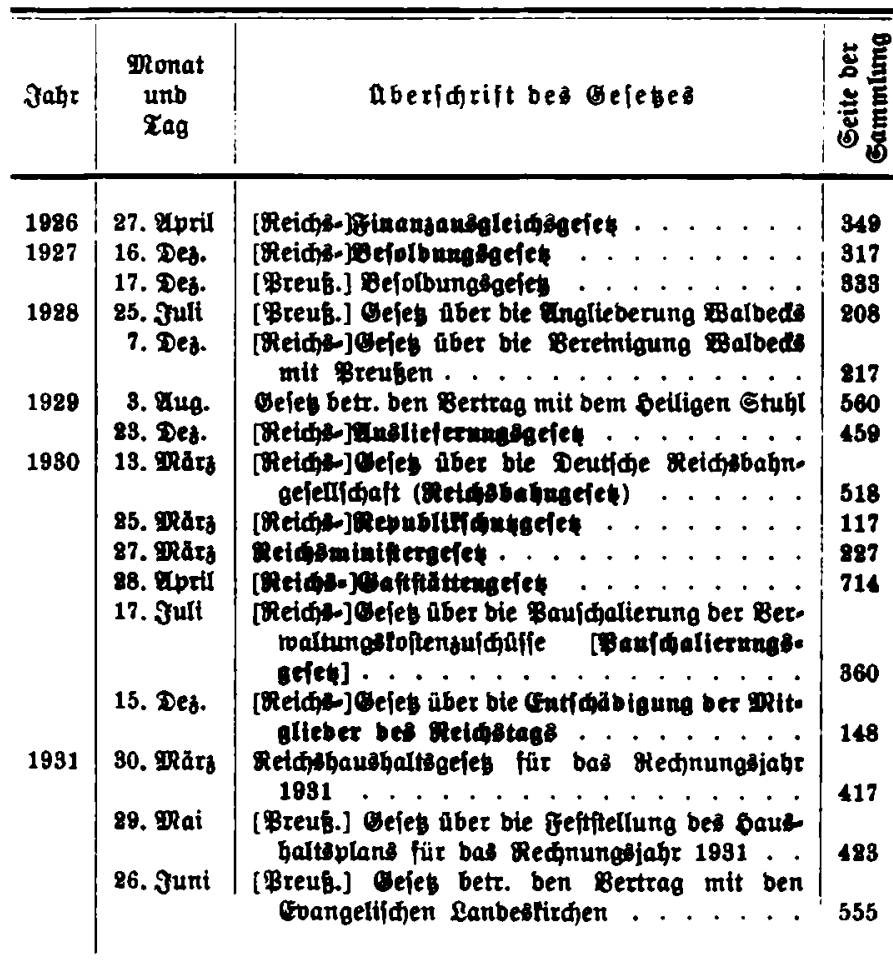


